\title{
A Protocol for a Systematic Review of Economic Evaluation Studies Conducted on Influenza and Recent Pandemic Prevention and Control
}

\author{
Amanuel Yigezu ( $\nabla$ yigezuamanuel@yahoo.com ) \\ Disease Control Priorities - Ethiopia https://orcid.org/0000-0003-2792-2163 \\ Mezgebu Mengistu \\ University of Gondar
}

Alemnesh Mikuzie

Ethiopian Public Health Institute

\section{Study protocol}

Keywords: Pandemic, COVID-19, Influenza, economic evaluation, Public health measures

Posted Date: July 23rd, 2020

DOI: https://doi.org/10.21203/rs.3.rs-46767/v1

License: (c) (i) This work is licensed under a Creative Commons Attribution 4.0 International License.

Read Full License 


\section{Abstract}

Background: The COVID-19, illness caused by a novel coronavirus, is the cause of an outbreak of respiratory illness in Wuhan, Hubei Province China beginning in 31 December 2019. The direct and indirect impacts of the pandemic and the public health measures taken to prevent the distribution of the pandemic on mortality and morbidity, and social and economic status of the community are enormous.

The public health measures against the pandemic such as school closure, unemployment and business shutdown incur cost on the society; reduce the provision and quality of health services due to the diversion of resource allocation to COVID-19 response (prevention and control) activities; result in negative influence on the healthcare seeking behavior of the society; and increase cost of seeking healthcare due to reduced global pharmaceutical supply chain. Evidence-based decision is crucial to prevent and manage the catastrophic impact of a pandemic and its public health measures. The aim of this review is to systematically review economic evaluation of interventions to respond to coronavirus pandemics in the global perspective. We included researches on influenza pandemic and COVID-19 as there are only few studies conducted in COVID-19.

Method: A full and partial economic analysis conducted on influenza and COVID-19 pandemic published in English language between 2012 and 2020 will be eligible. Studies with and without comparators will be included. We will build an exhaustive database search strategy in the global health cost-effectiveness analysis (CEA) registry, PubMed, African Journals online and Elsevier for relevant studies. We will do a hand-searching of cited research and bibliographies of all identified relevant studies. Two authors will independently select potentially eligible titles and abstracts, extract data from selected studies using structured collection form after a pre-test and do a risk of bias assessment. Third author will be involved if there is disagreement between the two. If variation of extracted data between the reviewers is observed, the phase will be repeated, and if discrepancies between data extractors continued, third reviewer will be involved. We will use a narrative summary and tables to summarize the result and characteristics of eligible studies. In addition, attempts will be made to change reports in case and death averted to disability life year averted (DALY) averted.

Discussion: Unless a careful decision is made to control a pandemic; the health, economy, social, and political aspect of countries will be in impacted. Public health measures that are implemented to respond to a pandemic should be cost-effective. This review will systematically analyze economic evaluation studies conducted on influenza and COVID-19 pandemics and synthesize evidence for public health measures. This review will further identify existing gaps in research for more studies.

\section{Systematic review registration: PROSPERO: CRD42020192384}

\section{Background}

The coronavirus disease (COVID-19), an illness caused by a novel coronavirus, is the cause of an outbreak of respiratory illness in Wuhan, Hubei Province, China beginning in 31 December 2019. The 
world health organization has declared it a public health emergency of international concern on 11, March 2020 [1, 2]. The virus has higher levels of transmissibility and pandemic risk than SARS-COV and MERS-COV [3] with a reproduction number between 2 and 2.5 [1]. The virus is primarily transmitted between people through respiratory droplets and contact routes. Fever, sore throat, flu-like symptoms such as coughing, shortness of breath and fatigue are the symptom of the disease.

The impact of the pandemic is beyond the health sector; it also affects the economic, political, and social dimensions of the countries [4]. The COVID-19 pandemic and the public health measures to respond to it have led to a substantial economic shock other than its impact on the morbidity and mortality caused by the virus. The number of COVID-19 cases reached around 5.8 million with around 358,000 death tolls as of 28th May 2020. During the same period, USA, Brazil, Russia, Spain, and UK were the top five countries accounting for more than half (53.2\%) of the total number of the COVID 19 cases; and USA, UK, Italy, France and Spain were the top five countries accounting for more than three-fifth (63.\%) of total death tolls due to the COVID 19 pandemic. In Africa, as of 28th May 2020, around 127,000 coronavirus cases, and 3,800 deaths were reported; and South Africa, Egypt, Algeria, Nigeria, and Morocco were top five countries in terms of the COVID 19 cases whereas Egypt, Algeria, South Africa, Nigeria and Morocco were the top five countries in terms of death tolls. Public health measures such as resource allocations to COVID-19 prevention and control activities (mass testing, treatment, and quarantine), physical distancing, movement restriction, city lockdown, business and industry shutdown, and school closure have been taken by countries. For example, China has successfully slowed down the spread of the virus through lockdown of high risk areas [5]. However, the cost associated with city lockdown is very high. For example, when Tokyo was lockdown for a month, Japan lost 27 trillion yen, 5.3\% of its annual GDP [6]. Due to job losses, the increase in inequality between the rich and the poor might lead to crime by the poor to secure their needs [7]. School closure would increase time lost from work (productivity loss) for families and represented higher monthly household expense, especially for community with low socioeconomic status [8-10]. Food insecurity [11], mental health problem and suicide [12,13], and death from other diseases also increase[14]. In addition, the governments from developing countries might be forced to borrow[14], and faced with increased rate of unemployment and violence which can lead to lack of faith in the government by the community thereby lead to political instability [15].

The world is always at risk of a pandemic at a certain time. For example, it is assumed that a global influenza pandemic can occur every 10 to 50 years interval [16]. study also indicated that over 62 million deaths mostly from developing countries would have occurred globally had there been a pandemic event in 2004 [17]. Strong health system led by evidence based public health measures minimizes potential damage occurring to the community. The use of resources in an efficient way is one area where policy makers need evidence. Because resources are scarce, policy makers need to prioritize their measures in a way that will save lives of most of the community. Decision makers can use the information from this finding to prioritize public health measures needed to be implemented at the time of a pandemic. We found two previously conducted systematic reviews on economic evaluation of pandemic preparedness plan. However, they included studies conducted up to $2014[18,19]$. The aim of this review is to take lessons from economic evaluation of different studies conducted between 2012 and 2020 on influenza 
pandemic and recent studies on COVID-19. Our findings will contribute to the better understanding of the economic evaluation of pandemic interventions and will be useful in the planning for and response to current and future pandemics.

\section{Method}

We used the Preferred Reporting Items for Systematic Review and Meta-Analyses (PRISMA) Protocol guidelines to prepare the protocol, and the finding will also be reported in accordance with PRISMA statement [20]. The completed PRISMA-P checklist for our protocol is attached as supporting file [supplementary file 1]

\section{Operational definition}

\section{Pandemic}

epidemic occurring worldwide, or over a very wide area, crossing international boundaries and usually affecting a large number of people [21].

\section{Influenza}

a contagious, acute respiratory illness caused by influenza virus [16]

COVID-19

A new strain of coronavirus that has not been previously identified in humans.

\section{Full economic evaluation}

A type of economic evaluation that needs identification, measurement, valuation, and comparison of both costs (inputs) and consequences (outputs, outcomes) of alternative courses of actions [22].

\section{Criteria for eligibility of studies}

\section{Inclusion criteria}

Full economic evaluation model-based or trial-based studies conducted in English will be included. They include cost-minimization, cost-effectiveness, cost-utility, and cost-benefit analyses [22, 23].

\section{Exclusion criteria}

Economic evaluation studies conducted before 2012 will be excluded. Studies reporting only health outcomes but not costs will not be included. We will check eligibility of bibliographies of systematic reviews, but not the review itself. Commentaries, reviews, or methodological articles, and editorials will also be excluded. 


\section{Population, intervention, comparator, and outcome (PICO)}

Population $(\mathrm{P})$ : The population of interest will be all populations impacted with the pandemic causing respiratory diseases (influenza and COVID-19). Intervention type (I): Any intervention related to public health measures to prevent and treat influenza and COVID-19 pandemic will be eligible for inclusion. Comparison (C): Can include an alternative standard of care (existing strategy) or a do-nothing scenario. Outcome measures (0): Outcomes will include measures related to cost-effectiveness ratio, cost-utility and cost benefit analysis associated with the public health measures compared. The full economic evaluation report of "Incremental Cost-Effectiveness Ratio (ICER) and Average Cost-Effectiveness Ratio (ACER), Cost-Benefit Ratio (CBR), and net costs will be recorded. All measures effectiveness including, Quality Adjusted Life Year (QALY), Disability Adjusted Life Year (DALY), Life Year Gained (LYG), cases averted, death averted, will be included in the CEA and CUA.

\section{Search methods for identification of studies}

We will build an exhaustive database search strategy in the global health cost-effectiveness analysis (CEA) registry, PubMed, African Journal online and Elsevier for relevant studies according to the "Centre for Reviews and Dissemination's (CRD) Guidance" recommendations [24]. We will do a manual-searching of reference sections of all identified relevant studies and also search for cited references. In addition, relevant internet sites will also be searched for any useful findings. We will first develop multiple search terms and then we will combine the search terms using "OR" and "AND". Filters will also be used to limit the search to "Humans" and "English". The search strategy to be used on PubMed is as follows:(cost adj analysis[Title/Abstract]) OR (expenditure* not energy[Title/Abstract]) OR (economic*[Title/Abstract]) OR price[Title/Abstract] OR pricing[Title/Abstract]) OR (value for money[Title/Abstract]) OR (budget* [Title/Abstract]) OR (return on investment[Title/Abstract]) OR (value proposition[Title/Abstract]) OR (markov chains[Title/Abstract])) AND((school*[Title/Abstract]) OR (city*[Title/Abstract]) OR (Country[Title/Abstract] OR (Quarantine[Title/Abstract]) OR (mass testing[Title/Abstract]) (Vaccine* [Title/Abstract]) OR (clos*[Title/Abstract]) OR (travel*[Title/Abstract]) OR (trade*[Title/Abstract]) OR (surveillance*[Title/Abstract]) OR (emergency*[Title/Abstract]) OR (restrict*[Title/Abstract]) OR (social* [Title/Abstract]) OR (isolation[Title/Abstract]) (Stockpiling*[Title/Abstract]) OR *[Title/Abstract]) OR (physical*[Title/Abstract]) OR (Pharmaceutical[Title/Abstract]) OR (drug*[Title/Abstract]) OR (personal* [Title/Abstract]) OR (antiviral*[Title/Abstract]) OR (hospital*[Title/Abstract]) OR (Hygiene [Title/Abstract]) OR (food* [Title/Abstract]) AND ((Pandemic*[Title/Abstract]) OR (Influenza[Title/Abstract]) OR (H1N1[Title/Abstract]) OR (COVID-19[Title/Abstract]) OR (Flu[Title/Abstract])).

We may modify and try changing search strategies across the databases and we will only search for studies written in English. We will use EndNote $x 6$ to manage search results and remove duplicate records and we will also use Microsoft excel to select studies and extract data.

\section{Selection of studies}


Two authors (AY and MM) will select potentially eligible titles independently. In addition, the full text of the selected titles will be collected by the two authors. Discussion will be conducted on the selected title between the two authors. The authors will do second stage screening if titles are approved by either of the authors. Disagreements with respect to including eligible studies will be approved by discussion in the presence of a third author (AM).

\section{Data extraction and management}

Two authors will extract data from selected studies independently using structured data collection form on Microsoft Excel after a pre-test. we will structure the form based on the "Consolidated Health Economics Evaluation Reporting Standards (CHEERS)" format [25] and NHS Economic Evaluation Database (NHS EED) guidelines [26]. It will include details of information on the setting, the year of conducted study, methods, perspectives, discounting, costing year, outcome measures or findings, and risk of bias. Authors will discuss and compare the extracted data and make further improvement. Third author (AM) will involve if there is disagreement between the two authors. Direct communication with authors will also be made for missing data. Supporting file is attached in for detail of the data extraction form [supplementary file 2].

\section{Risk of bias assessment}

The authors will do a risk of bias assessment using recommended tools for health economic studies. The two authors (AY and MM) will independently assess the risk of bias for methodological quality using CHEERS statement [25]. The third author will review (AM) the works of the two reviewers if there is any disagreement. The finding of these appraisals will be discussed in the narrative synopsis at the end.

\section{Data analysis and synthesis}

Description of the characteristics of the studies and results will be displayed using narrative summary and tables. The narrative summary will be structured by type of public health measure or intervention, and type of economic evaluation, and country of study. The price year and the currency will be reported and we will try to change it to 2019 price year using appropriate conversion factors [27]. Attempts will be made to convert the findings of studies reporting their cost-effectiveness analysis result in cost per death averted and cost per case averted to cost per DALY. For CBA studies, we will extract their report on cost, number of cases and deaths avoided to estimate the cost-effectiveness ratio. In addition, parameters in the cost-effectiveness analysis examined for uncertainty in the cost-effectiveness ratio estimates will be reported.

\section{The Quality Of Economic Evidence Assessment}

We will try to assess the quality of economic evidence using the five domains recommended by the "Grading of Recommendations Assessment, Development, and Evaluation" (GRADE) approach [28].

\section{Discussion}


Pandemics are known to have an immediate and intergenerational health and non-health impact. For, example the 1918 influenza pandemic had not only taken millions of lives, but also affected the social, economic and health aspects of next generation [29].Therefore, public health response to avert a potential loss due to a pandemic will not only saves lives now, it also improves recovery of the health system and other sectors. Since resources are scarce, information on prioritizing resources at the time of a pandemic to save many lives is crucial to decision makers. Evidence with economic evaluation of pandemic prevention and control mechanisms will guide decision maker to prepare an effective pandemic preparedness plan, take appropriate action at the time of a pandemic, and save the cost on future generations. This systematic review will aid decision makers with economic evaluation of interventions. This review will further identify existing gaps in research for more studies. Our findings will be published in a peer-review journal and subsequently disseminated to policy-makers through conferences and stakeholder meetings.

\section{Supplementary file 1}

PRISMA-P 2015 Checklist. PRISMA-P checklist indicating the list of information found in the protocol.

\section{Supplementary file 2}

Draft of data extraction format

\section{Abbreviations}

ACER

Average Cost-Effectiveness Ratio; CBA:Cost-Benefit Analysis; CEA:Cost-Effectiveness Ratio; CHEERS:Consolidated Health Economic Evaluation Reporting Standards; CRD:Centre for Reviews and Dissemination; DALY:Disability Adjusted Life year; GRADE:Grading of Recommendations Assessment, Development, and Evaluation; ICER:Incremental Cost-Effectiveness Ratio; LYG:Life year Gained; NHS EED:NHS Economic Evaluation Database; QALY:Quality Adjusted Life Year

\section{Declarations}

\section{Acknowledgment}

We thank the national Data management center (NDMC) at the Ethiopian Public Health Institute (EPHI) and the Health Intervention and Technology Assessment Program (HITAP) for their contribution. The HITAP has supported us in providing trainings and comments on the protocol.

Funding: This research received no specific grant from any funding agency in the public, commercial, or not-for-profit sectors

Availability of data and material: The data that support the findings of this study are available from the corresponding author on reasonable request. 
Authors' contributions

$\mathrm{AY}$ is the guarantor of the review. All authors participated in conception of the research idea and review of the manuscript. MM and AM developed the search strategies. AY conducted the preliminary searches. All authors prepared data extraction form and drafted the protocol and approved the final version of the manuscript.

Ethics approval and consent to participate: Not applicable.

Consent for publication: Not applicable

Competing interests: The authors declare that they have no competing interests

\section{References}

1. Evans O: Socio-economic impacts of novel coronavirus: The policy solutions. 2020.

2. Adhikari SP, Meng S, Wu Y-J, Mao Y-P, Ye R-X, Wang Q-Z, Sun C, Sylvia S, Rozelle S, Raat H, Zhou H: Epidemiology, causes, clinical manifestation and diagnosis, prevention and control of coronavirus disease (COVID-19) during the early outbreak period: a scoping review.Infectious Diseases of Poverty 2020, 9:29.

3. Liu T, Hu J, Kang M, Lin L, Zhong H, Xiao J, He G, Song T, Huang Q, Rong Z, et al: Transmission dynamics of 2019 novel coronavirus (2019-nCoV). 2020.

4. Acikgoz O, Gunay A: The early impact of the Covid-19 pandemic on the global and Turkish economy.Turk J Med Sci 2020.

5. Lau H, Khosrawipour V, Kocbach P, Mikolajczyk A, Schubert J, Bania J, Khosrawipour T: The positive impact of lockdown in Wuhan on containing the COVID-19 outbreak in China.J Travel Med 2020.

6. Inoue H, Todo Y: The Propagation of the Economic Impact through Supply Chains: The Case of a Mega-City Lockdown against the Spread of COVID-19.SSRN Journal SSRN Electronic Journal 2020.

7. The Times of India: An outline cost-benefit test of COVID-19 lockdowns. In The Times of India. New Delhi: Times of India Pr.; 2020.

8. Brown ST, Tai JH, Bailey RR, Cooley PC, Wheaton WD, Potter MA, Voorhees RE, LeJeune M, Grefenstette JJ, Burke DS: Would school closure for the 2009 H1N1 influenza epidemic have been worth the cost?: a computational simulation of Pennsylvania.BMC public health 2011, 11:353.

9. Borse RH, Behravesh CB, Dumanovsky T, Zucker JR, Swerdlow D, Edelson P, Choe-Castillo J, Meltzer MI: Closing schools in response to the 2009 pandemic influenza A H1N1 virus in New York City: economic impact on households. Clin Infect Dis 2011, 52 Suppl 1:S168-172.

10. Basurto-Davila R, Garza R, Meltzer MI, Carlino OL, Albalak R, Orellano PW, Uez O, Shay DK, Santandrea C, del Carmen Weis M, et al: Household economic impact and attitudes toward school closures in two cities in Argentina during the 2009 influenza A (H1N1) pandemic.Influenza Other Respir Viruses 2013, 7:1308-1315. 
11. Keogh-Brown MR, Smith RD: The economic impact of SARS: How does the reality match the predictions? Health Policy 2008, 88:110-120.

12. Zhang Y, Ma ZF: Impact of the COVID-19 Pandemic on Mental Health and Quality of Life among Local Residents in Liaoning Province, China: A Cross-Sectional Study.Int J Environ Res Public Health 2020, 17.

13. Gao J, Zheng P, Jia Y, Chen H, Mao Y, Chen S, Wang Y, Fu H, Dai J: Mental health problems and social media exposure during COVID-19 outbreak.PLoS One 2020, 15:e0231924.

14. Seidou Sanda I, Uneca, Kedir A, Lom A, Diouf A, Acosta C, Ngonze C, Ikome F, Zulu J, Namegabe J-L, et al: Socio-economic Impacts of Ebola on Africa. 2015.

15. Costa K: THE CAUSE OF PANIC AT THE OUTBREAK OF COVID-19 IN SOUTH AFRICA - A COMPARATIVE ANALYSIS WITH SIMILAR OUTBREAK IN CHINA AND NEW YORK. 2020.

16. Potter CW: A history of influenza.Journal of applied microbiology 2001, 91:572-579.

17. Murray CJL, Lopez AD, Chin B, Feehan D, Hill KH: Estimation of potential global pandemic influenza mortality on the basis of vital registry data from the 1918\&\#x2013;20 pandemic: a quantitative analysis. The Lancet 2006, 368:2211-2218.

18. Velasco RP, Praditsitthikorn N, Wichmann K, Mohara A, Kotirum S, Tantivess S, Vallenas C, Harmanci $\mathrm{H}$, Teerawattananon $\mathrm{Y}$ : Systematic review of economic evaluations of preparedness strategies and interventions against influenza pandemics.PloS one 2012, 7.

19. Pasquini-Descomps H, Brender N, Maradan D: Value for Money in H1N1 Influenza: A Systematic Review of the Cost-Effectiveness of Pandemic Interventions. Value Health 2017, 20:819-827.

20. Moher D, Shamseer L, Clarke M, Ghersi D, Liberati A, Petticrew M, Shekelle P, Stewart LA, Group P-P: Preferred reporting items for systematic review and meta-analysis protocols (PRISMA-P) 2015 statement.Systematic Reviews 2015, 4:1.

21. Kelly $\mathrm{H}$ : The classical definition of a pandemic is not elusive.Bulletin of the World Health Organization 2011, 89:540-541.

22. Cochrane handbook for systematic reviews of interventions. In incorporating economic evidence. Hoboken, N.J.: Wiley; 2011: 449-480

23. Teerawattananon $Y$, Russell $S$, Mugford $M$ : A systematic review of economic evaluation literature in Thailand: are the data good enough to be used by policy-makers? Pharmacoeconomics 2007, 25:467479 .

24. Centre for R, Dissemination: CRD's guidance for undertaking reviews in healthcare. York: York Publ. Services; 2009.

25. Husereau D, Drummond M, Petrou S, Carswell C, Moher D, Greenberg D, Augustovski F, Briggs AH, Mauskopf J, Loder E: Consolidated Health Economic Evaluation Reporting Standards (CHEERS) statement.eurojhealecon The European Journal of Health Economics 2013, 14:367-372.

26. Craig D, Rice S: NHS economic evaluation database handbook. York: Centre for Reviews \& Dissemination, University of York; 2007. 
27. Turner HC, Lauer JA, Tran BX, Teerawattananon Y, Jit M: Adjusting for Inflation and Currency Changes Within Health Economic Studies. Value in Health 2019, 22:1026-1032.

28. Brunetti M, Shemilt I, Pregno S, Vale L, Oxman AD, Lord J, Sisk J, Ruiz F, Hill S, Guyatt GH, et al: GRADE guidelines: 10. Considering resource use and rating the quality of economic evidence.J Clin Epidemiol 2013, 66:140-150.

29. Cook CJ, Fletcher JM, Forgues A: Multigenerational Effects of Early-Life Health Shocks.Demography 2019, 56:1855-1874.

\section{Supplementary Files}

This is a list of supplementary files associated with this preprint. Click to download.

- supplementaryfile2.docx

- PRISMAChecklistProtocol.docx 